\title{
LA ORIENTACIÓN AL MERCADO COMO ESTRATEGIA DE EMPRENDIMIENTO EN EL ISTMO OAXAQUEÑO
}

Felipe de Jesús Cruz Celis Martha Lis Garrido Cardona

Mario Rojas Miranda

RECIBIDO: 13 DE SEPTIEMBRE DE 2017 ACEPTADO: 12 DE ENERO DE 2018.

\section{RESUMEN}

Tos resultados de las 384 encuestas aplicadas a los consumidores de jitomate de las poblaciones de Ixtepec, Juchitán, Tehuantepec y Salina Cruz, en la región del Istmo Oaxaqueño, México, son los siguientes: en cuanto a consumidores, el $73.06 \%$ compra jitomate Saladet; el $36.6 \%$ compra cada dos días; el 84.87 $\%$ compra de 1 a 2 kg/semana; el $79.34 \%$ compra el jitomate en el mercado local; el $62.73 \%$ lo utiliza para guisar; el 54.98 $\%$ dice que la principal característica de compra que busca es su precio. En cuanto a los proveedores, los resultados de las 73 encuestas aplicadas son los siguientes: el $40 \%$ del jitomate lo traen de Oaxaca, el $91.94 \%$ de los consumidores compra Saladet, el $48.39 \%$ vende más de $21 \mathrm{~kg}$ diarios, el $56.45 \%$ fija el precio con base en los costos, el $59.68 \%$ se abastece cada tercer día.

Palabras clave: Emprendedor, valor del cliente, orientación de mercado. 
Abstract

The results of 384 surveys applied in the cities of Ixtepec, Juchitán, Tehuantepec and Salina Cruz in the Isthmus of Tehuantepec region of the state of Oaxaca, Mexico yielded the following results: 73.06 $\%$ of the respondents buy $\mathrm{Sa}$ ladet tomatoes; $36.6 \%$ buy them every other day; $84.87 \%$ buy from one to two kilos per week; $79.34 \%$ buy tomatoes at the local market; $62.73 \%$ use them to cook and $54.98 \%$ say that the price is their foremost consideration when making a purchase. With respect to suppliers, the results of 73 surveys are as follows: $40 \%$ of tomatoes come from Oaxaca City, Oaxaca; $91.94 \%$ of consumers bought Saladet; 48.39 $\%$ sell more than 21 kilograms daily; $56.45 \%$ set prices based on cost; $59.68 \%$ supply themselves with tomatoes every third day.

Key words: Entrepreneurship, client's value, market orientation.

\section{INTRODUCCIÓN}

lo largo de la historia,
existen noticias sobre
numerosas hambru- nas que causaron millones de muertes en todos los continentes. La peor de ellas fue la que, acompañada de la plaga bubónica, causó alrededor de 43 millones de muertes en Europa entre los años 1345 y 1348. La más mortífera del siglo XX tuvo lugar en el norte de China y causó unos 30 millones de muertos después del "Gran salto adelante" de Mao, entre 1958-1962 (Pérez, 2000). En las últimas décadas del siglo XX, la hambruna se ha concentrado en África Subsahariana. En esta región, se estima que el hambre afectará a 40 millones de niños en el 2020 (Pinstrup, 1998).

En 1974 se celebró la Conferencia Mundial sobre Alimentación, en Roma, para discutir la crisis alimentaria mundial. El tema más importante ahí tratado fue la seguridad alimentaria, ya que los precios de los productos agrícolas se habían elevado a niveles sin precedentes y las existencias de grano eran excesivamente bajas. Se acrecentaron los temores de que el mundo se estaba dirigiendo en forma irrevocable hacia una escasez de alimentos, atribuible a cambios climatológicos desfavora- bles de largo plazo y a los elevados índices de crecimiento de la población (Siamwalla y Valdés, 1980). En este contexto, surgió el concepto de Seguridad Alimentaria Nacional (san) bajo el enfoque demográfico. La san se definió como la disponibilidad segura del suministro de alimentos suficientes para satisfacer las necesidades de consumo per cápita de un país en todo momento.

Amartya Sen, al analizar las hambrunas de Bengala de 1943 y de Etiopía en 1972 1974, comprobó que estas no se debieron a la falta de alimentos, ya que en ambos casos se exportaban desde las zonas afectadas hacia otras limítrofes o a la capital. Los comerciantes llevaban los alimentos guiados no por la necesidad de las personas, sino por la demanda que ejercían quienes tenían poder adquisitivo. Por consiguiente, confirmó que la causa de la hambruna radica en la incapacidad de las familias pobres para acceder a los alimentos, Sen las denominó titularidades (Pérez, 2000).

Los emprendedores conocen mucho acerca del producto o servicio que elaboran, pero en gran medida pueden 
carecer de un componente de conocimientos en el área comercial que les permita identificar los clientes que más valorarían su producto o servicio. Esta situación se observa más en emprendimientos iniciados en unidades tecnológicas que surgen de investigación y desarrollo. Se puede decir que los emprendedores se enamoran de su proyecto, lo que les dificulta ser objetivos al momento de evaluar su potencial comercial. En consecuencia, los esfuerzos comerciales no responden a una estrategia concreta, pero no por ineficacia o incompetencia, sino por ignorancia respecto de algunos temas (Scaliter, 2005).

Los emprendedores se distraen de pensar en el producto o servicio que los apasiona y moviliza, para orientarse de manera objetiva hacia el mercado potencial y los clientes que deberían ser contactados, elaborando una lista que permite pensar en aspectos tácticos comerciales. El emprendedor necesita ver su producto o servicio en perspectiva, de manera objetiva, intentando eliminar la subjetividad que proviene de la inmersión en el producto o servicio para eva- luar el verdadero potencial en cuanto a precio definido por el valor agregado y las posibilidades de contacto definidas por la permeabilidad.

La presente investigación tiene como objetivo realizar un estudio de mercado con el fin de conocer la demanda de los clientes, así como la oferta de los proveedores, para conocer la demanda y oferta del jitomate en el Istmo oaxaqueño de manera objetiva y evaluar el verdadero potencial del mercado del jitomate en cuanto a precio definido por el valor agregado.

Esta investigación se desarrolla en torno a las estrategias de afrontamiento porque actualmente, al contrario de lo que ocurría en décadas pasadas, los productores agrícolas deben adoptar técnicas innovadoras para tener ventaja, debido a la globalización de los mercados, el cambio en las necesidades y exigencias de los clientes, la rápida evolución en las tecnologías y la gran importancia del respeto al medio ambiente.

\section{MARCO TEÓRICO}

工 $\begin{aligned} & \text { a globalización y el en- } \\ & \text { torno altamente com- } \\ & \text { petitivo exigen a las }\end{aligned}$ empresas estar orientadas al mercado y gestionar su cartera de clientes como un activo estratégico clave para aumentar la rentabilidad del negocio y lograr una ventaja competitiva sostenible en el tiempo.

Al plantear la necesidad de que las empresas agroalimentarias adopten una estrategia de orientación al mercado (om), se está pretendiendo introducir el concepto de marketing. Uno de los motivos de esta actuación es que el nuevo marco competitivo mundial plantea nuevos retos para el sector agroalimentario. En la vertiente de producción se afronta una situación de excedentes en buena parte de la producción agroalimentaria, de aparición de nuevos competidores y, por tanto, de saturación de los mercados. Además, se debe considerar un consumo cada vez más sofisticado y con un mayor nivel de exigencia hacia el producto, con gustos en cambio permanente. El precio ha dejado de ser el factor determinante para 
dar paso a elementos propios de la diferenciación.

A finales de los años 80 el Marketing Science Institute empezó a conceder a la orientación al mercado un papel esencial en la disciplina del marketing. Se han sucedido numerosas formas de entender este concepto. La literatura sobre este tema tiende a concentrarse en torno a dos corrientes de pensamiento mayoritarias: la orientación al mercado actitudinal (Narver y Slater, 1990) y la orientación al mercado comportamental (Kohli y Jaworski, 1990).

De esta forma, los autores Narver y Slater (1990) conciben la orientación al mercado como un fenómeno relacionado con las normas y los valores culturales de una organización.

Esta perspectiva identifica la orientación al mercado no tanto como una conducta sino más bien como una actitud o cultura. Por este motivo, a esta primera corriente de investigación se le denomina habitualmente cultural o actitudinal. Así, Nerver y Slater (1990) consideran que la orientación al mercado es un constructo integrado por tres dimensiones: la orientación al cliente, la orientación a la competencia y la coordinación interfuncional.

En la revisión de la literatura, una de las hipótesis más recurrentes en los trabajos especializados en la materia ha sido el tipo de relación existente entre orientación al mercado y resultados empresariales. Diversos son los trabajos que analizan dicha hipótesis (Deng y Dart, 1994; Pelham y Wilson, 1996; Ruekert, 1992) y argumentan la existencia de una relación positiva entre la orientación al mercado y el rendimiento empresarial. De este modo Langerak et al. (1997) hacen una comparación de las escalas de orientación al mercado más utilizadas y su relación con los resultados obtenidos. Llegan a la conclusión de que hay suficientes resultados empíricos para afirmar que la orientación al mercado influye positivamente en los resultados empresariales.

En este contexto, muchas compañías están tomando conciencia de que cada vez son menos rentables las estrategias dirigidas a mercados masivos. Por lo tanto, se torna necesario gestionar las relaciones con sus clientes de manera personalizada, desde una perspectiva del valor y de la rentabilidad a lo largo del ciclo de vida de las relaciones entre el cliente y la empresa, para obtener una ventaja competitiva sostenible en el tiempo.

Low (2005) ha corroborado en sus estudios que la orientación al mercado como filosofía de negocio actúa como generadora de ventajas competitivas y mejores resultados empresariales. Esto, porque una empresa orientada al mercado utiliza el conocimiento de este para la formulación e implementación de su estrategia (Morgan y Strong, 1998) y se destaca frente a sus competidores por su mayor habilidad para comprender, captar y retener a los clientes. La capacidad de comprensión del mercado permite a la empresa anticiparse a las oportunidades del mismo, y hace referencia a la habilidad para percibir y actuar sobre los eventos y tendencias del mercado (Kohli y Jaworski, 1990). La capacidad de relación con los clientes se refiere a la habilidad de las empresas para establecer, fortalecer y desarrollar relaciones rentables con el cliente (Grönroos, 1989). 
Al centrarse en el cliente, este pasa a estar por delante de los procesos internos de la organización, siendo lo prioritario la forma en que el cliente quiere interactuar con ella. De esta manera, el actuar para el cliente pasa a ser sustituido por actuar con el cliente. Por consiguiente, el enfoque va más allá de un marketing relacional, apuntando a un marketing colaborativo entre cliente y empresa para la creación de valor.

Porter (1985) sostiene que el concepto de ventaja competitiva surge básicamente del valor que una empresa es capaz de crear para sus compradores, el cual debe exceder al costo incurrido por la empresa para crear tal valor. El valor es lo que los compradores están dispuestos a pagar, y el valor superior surge por ofrecer precios más bajos que los competidores con beneficios equivalentes o por otorgar beneficios únicos que justifiquen un precio mayor. Según Grant (1995) una empresa posee una ventaja competitiva frente a sus competidores cuando tiene un potencial para obtener una tasa de rentabilidad mayor.

No necesariamente la ventaja competitiva se debe ver reflejada únicamente en una rentabilidad superior, ya que la empresa puede reducir beneficios y ganar cuotas de mercado o recompensar a sus empleados o ejecutivos. Por otro lado, los beneficios pueden ser invertidos en la adquisición de nuevos clientes valiosos para el negocio de la empresa.

Para determinar el valor real que genera la lealtad de los clientes, es primordial ir más allá de las características de los productos y servicios; es necesario buscar el aporte en la vida del cliente y en cómo la experiencia que rodea a la oferta adiciona o disminuye valor. El valor representa no lo que el producto o servicio hace, sino el impacto que tiene en la vida del cliente. Por lo tanto, el enfoque empresarial y de marketing debe centrarse primordialmente en el valor del tiempo de vida del cliente (Hogan et al., 2002).

La om surgió como una respuesta competitiva tendiente a crear nexos con los usuarios de los productos; se buscaba potencializar el número de transacciones que se realizaban con cada cliente, sin tener en cuenta la noción del tiempo y la visión a largo plazo. Pero la evolu- ción del mercadeo ha movido su referente desde el contexto transaccional convencional hacia el plano relacional, donde se persigue establecer nexos a futuro con los clientes para así garantizar la fidelidad, la rentabilidad y la lealtad de los mismos (Anderson, Fornell y Mazvancheryl, 2004).

Dicha evolución del mercadeo ha conllevado un aumento en la apropiación de recursos hacia esa área (Stewart, 2008) que, aunada a la búsqueda de la eficiencia en el manejo de fondos, presiona a las directivas de las compañías y a sus departamentos de mercadeo para que revisen las inversiones desde la perspectiva financiera (Wills y Webb, 2007).

México enfrenta problemas alimentarios preocupantes. Hay problemas en la producción, el almacenamiento, la distribución y la comercialización de alimentos. Por otra parte, existen grandes oportunidades para la venta de hortalizas, ya que la misma gente de las comunidades demanda productos frescos de calidad y a buen precio. Pero para lograr lo anterior se requiere generar un proceso gradual de cambios tecnológicos, que inician 
Gráfica 1. Superficie cosechada en Oaxaca

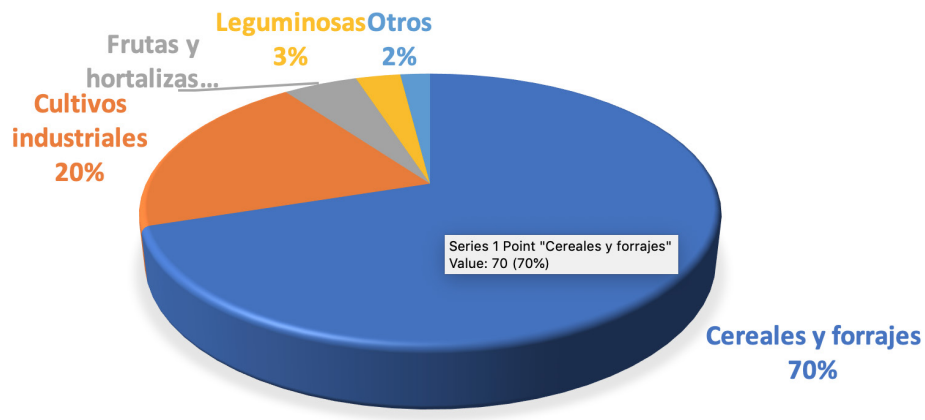

Fuente: Subsecretaría de Fomento a los Agronegocios (sfa, 2011).

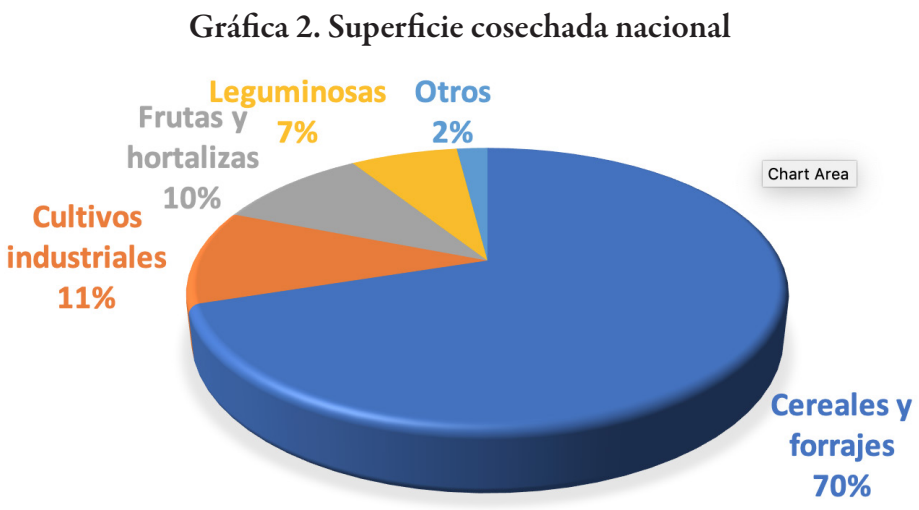

Fuente: Subsecretaría de Fomento a los Agronegocios (sfa, 2011).

con el cultivo de hortalizas. A continuación, se presentan la distribución de la superficie cosechada en Oaxaca en 2011 (gráfica 1) y la distribución de la superficie cosechada a nivel nacional (gráfica 2), de acuerdo con la Subsecretaría de Fomento a los Agronegocios (sfa, 2011):

La distribución de la superficie cosechada a nivel nacio-

var en la gráfica 2 . cado y así tener ventajas competitivas.

\section{Metodología}

\section{Diseño DE} INSTRUMENTO DE MEDICIÓN

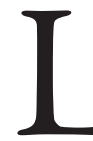

a investigación se desarrolló con enfoque cuantitativo; se diseñó un instrumento de medición para la recolección de datos en los municipios de Salina Cruz, Tehuantepec, Juchitán e Ixtepec, Oaxaca, México, que permitiera extraer información bajo el esquema del muestreo estratificado. Los reactivos se definieron con base en el objetivo establecido.

\section{Selección de la} MUESTRA

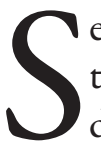

Comparando la superficie nacional cosechada con la del estado de Oaxaca, hay una diferencia de producción del 50 $\%$. por lo tanto, es necesario desarrollar nuevos emprendimientos con el fin de conocer a los clientes, para establecer estrategias orientadas al merser representativo de la misma, y de la cual se extrajeron los datos para responder a las preguntas de investigación y llegar al objetivo central, generando nuevo conocimiento (Hernández, Fernández y Baptista, 2010). En la investigación, la población o universo están representados por las cuatro ciu- 
Tabla 1. Número de mujeres mayores de 20 años por población

\begin{tabular}{cccc}
\hline Población & $\begin{array}{c}\text { Número de mu- } \\
\text { jeres }\end{array}$ & $\begin{array}{c}\text { Porcentaje del } \\
\text { total }\end{array}$ & $\begin{array}{c}\text { Población } \\
\text { muestra }\end{array}$ \\
\hline Ixtepec & 9020 & 9.8 & 38 \\
\hline Juchitán & 30145 & 32.8 & 126 \\
\hline Tehuantepec & 24687 & 26.8 & 103 \\
\hline Salina Cruz & 28007 & 30.4 & 117 \\
\hline Totales & 91859 & 100.0 & 384 \\
\hline
\end{tabular}

Fuente: Elaboración propia.

Tabla 2. Número de proveedores registrados en el Municipio por población

\begin{tabular}{|c|c|c|c|}
\hline Población & $\begin{array}{c}\text { Número de } \\
\text { distribuidores }\end{array}$ & $\begin{array}{c}\text { Porcentaje del } \\
\text { total }\end{array}$ & Población muestra \\
\hline Ixtepec & 12 & 16.4 & 12 \\
\hline Juchitán & 25 & 34.2 & 25 \\
\hline Tehuantepec & 16 & 21.9 & 16 \\
\hline Salina Cruz & 20 & 27.3 & 20 \\
\hline Totales & 73 & 100.0 & 73 \\
\hline
\end{tabular}

Fuente: Elaboración propia.

dades más pobladas del Istmo oaxaqueño; estas son: Ixtepec, Juchitán, Tehuantepec y Salina Cruz; para poder determinar el tamaño de la muestra se aplicó al número de mujeres mayores de veinte años que habitan estas poblaciones según el censo 2010 de inegi y se preguntó, en la Regiduría de la Secretaría de Hacienda y Crédito Público de cada municipio por el número de verdulerías registradas. A estos establecimientos se les nombró proveedores. Como paso siguiente, se procedió a determinar el tamaño de la muestra.

El tipo de muestreo empleado fue probabilístico, en donde todos los elementos de la población tienen la misma posibilidad de ser elegidos. La ventaja es que puede medirse el tamaño del error en las predicciones y su objetivo se centra en reducirlo al mínimo; al resultado se le denomina error estándar (Hernández et al., 2010); en este proyecto de investigación, el error fue de 5 $\%$, dejando así un nivel de con- fianza del $95 \%$. Para fines de la presente investigación, las unidades de observación (clientes y proveedores) fueron seleccionados en forma aleatoria, es decir, al azar, teniendo cada elemento la misma probabilidad de ser elegido.

El número de personas mayores de veinte años que representaron la población fueron 91859 personas. El cálculo se realizó mediante la siguiente ecuación para poblaciones finitas.

$\mathrm{N} \times \mathrm{Z}^{2} \alpha \times \mathrm{p} \times \mathrm{q}$

$\mathrm{N}=$

$\mathrm{d}^{2} \mathrm{x}(\mathrm{N}-1)+\mathrm{Z}^{2} \alpha \times \mathrm{p} \times \mathrm{q}$

En donde:

$\mathrm{N}=$ tamaño de la población; $\mathrm{Z}=$ nivel de confianza; $\mathrm{p}$ = probabilidad de éxito o proporción esperada; $\mathrm{q}=$ probabilidad de fracaso; $d=$ precisión (error máximo admisible en términos de proporción).

\section{Segmentación} DE LA INFORMACIÓN POR POBLACIÓN

e acuerdo al tamaño
de muestra, se realizó
un recálculo de los porcentajes asignados a cada 
Tabla 3. Resultados obtenidos de las encuestas a consumidores en el Istmo oaxaqueño

\begin{tabular}{|c|c|c|c|c|c|c|}
\hline Preguntas & OPCIONES & $\begin{array}{l}\text { Resultado en } \\
\text { \%, Ixtepec }\end{array}$ & $\begin{array}{l}\text { Resultado en } \\
\% \text {, Tehuante- } \\
\text { pec }\end{array}$ & $\begin{array}{l}\text { Resultado en } \\
\% \text {, Salina Cruz }\end{array}$ & $\begin{array}{l}\text { Resultado en } \\
\% \text {, Juchitán }\end{array}$ & $\begin{array}{l}\text { Total de los re- } \\
\text { sultados en } \%\end{array}$ \\
\hline \multirow{2}{*}{$\begin{array}{c}\text { 1. ¿Compra } \\
\text { usted jitomate? }\end{array}$} & Sí & 100 & 100 & 93 & 100 & 98.19 \\
\hline & No & 0 & 0 & 7 & 0 & 1.81 \\
\hline \multirow{3}{*}{$\begin{array}{c}\text { 2. ¿Qué tipo } \\
\text { de jitomate } \\
\text { compra? }\end{array}$} & Criollo & 5 & 19 & 21 & 22 & 17.34 \\
\hline & Saladet & 90 & 75 & 69 & 61 & 73.06 \\
\hline & Bola & 5 & 6 & 10 & 17 & 9.60 \\
\hline \multirow{4}{*}{$\begin{array}{c}\text { 3. ¿Con qué } \\
\text { frecuencia } \\
\text { compra usted } \\
\text { jitomate? }\end{array}$} & Diario & 20 & 35 & 22 & 38 & 29.15 \\
\hline & Cada 2 días & 30 & 42 & 36 & 32 & 36.06 \\
\hline & Cada semana & 48 & 21 & 40 & 26 & 32.21 \\
\hline & Quincenal & 2 & 3 & 1 & 4 & 2.58 \\
\hline \multirow{3}{*}{$\begin{array}{l}\text { 4. ¿Cuánto ji- } \\
\text { tomate compra } \\
\text { regularmente? }\end{array}$} & $1-2 \mathrm{~kg}$ & 82 & 85 & 88 & 85 & 84.87 \\
\hline & $3-4 \mathrm{~kg}$ & 15 & 10 & 9 & 8 & 10.33 \\
\hline & Más de $5 \mathrm{~kg}$ & 3 & 5 & 3 & 7 & 4.80 \\
\hline \multirow{4}{*}{$\begin{array}{c}\text { 5. ¿En qué } \\
\text { lugar acostum- } \\
\text { bra comprar } \\
\text { jitomate? }\end{array}$} & Mercado & 65 & 83 & 90 & 78 & 79.34 \\
\hline & Tianguis & 22 & 3 & 7 & 7 & 9.23 \\
\hline & Supermercado & 3 & 4 & 3 & 4 & 3.68 \\
\hline & Otro & 10 & 10 & 0 & 11 & 7.75 \\
\hline \multirow{3}{*}{$\begin{array}{l}\text { 6. ¿Qué uso le } \\
\text { da al jitomate? }\end{array}$} & Salsa & 6 & 26 & 30 & 33 & 24.72 \\
\hline & Ensalada & 17 & 10 & 10 & 14 & 12.55 \\
\hline & Guisado & 77 & 64 & 60 & 53 & 62.73 \\
\hline \multirow{4}{*}{$\begin{array}{c}\text { 7. ¿Qué carac- } \\
\text { terísticas toma } \\
\text { en cuenta al } \\
\text { comprar jito- } \\
\text { mate? }\end{array}$} & Precio & 73 & 43 & 48 & 58 & 54.98 \\
\hline & Tamaño & 3 & 5 & 3 & 6 & 4.43 \\
\hline & Sabor & 7 & 24 & 9 & 7 & 11.81 \\
\hline & Textura & 17 & 28 & 40 & 29 & 28.78 \\
\hline
\end{tabular}

Fuente: Elaboración propia. 
población estudiada, siguiendo el mismo procedimiento hecho con la población total, con lo cual se determinó el número de personas (tabla 1) y número de proveedores (tabla 2);

\section{LEVANTAMIENTO} DE LAS ENCUESTAS Y CODIFICACIÓN

ara llevar a cabo el le-
vantamiento de encues-
tas se procedió primero a capacitar a los encuestadores en la utilización del instrumento de evaluación; se realizó una prueba piloto y se procedió a identificar a la población objetivo con base base en la segmentación de la información por población.

\section{BASE DE DATOS}

a base de datos se dise-
nó y se elaboró en spss
versión 20 .

\section{Procesamiento}

DE LA

\section{INFORMACIÓN}

Pormación, se procedió ron y se agruparon los datos de acuerdo con las variables que se evaluaron con las diferen- tes preguntas que conforman el cuestionario. Se utilizaron medidas de tendencia central: media, mediana, moda. Se realizó un análisis por medio de pruebas estadísticas.

\section{ANÁlisis e INTERPRETACIÓN DE RESULTADOS \\ tewart (2008) señala que \\ la om es una de las últi- \\ $\bigcup$ mas fronteras salvajes en} los negocios americanos, donde vaqueros con ideas salvajes creaban compañías de la nada, destacando que el rompimiento de la burbuja terminó con estas viejas prácticas de gestión donde las áreas de mercadeo prometían resultados sin tener una manera de cuantificarlos, más allá de algunas mediciones blandas. Esto ha movido a practicantes y académicos de mercadeo para que asuman responsabilidad con los resultados que generan, demostrando cómo están creando valor (Jagpal, 2008). Luego, se han propuesto metodologías para llevar a términos financieros los resultados alcanzados con las estrategias y tácticas de mercadeo (Gupta y Lehmann, 2006), todas ellas encuadradas dentro de una filosofía general que en última instancia permita cuantificar el retorno de las inversiones de mercadeo y de una de sus principales inversiones, la publicidad (Briggs, 2006).

La tabla 3 presenta los resultados obtenidos de las encuestas realizadas a los consumidores, y la tabla 4, los resultados obtenidos de las encuestas a proveedores en el Istmo oaxaqueño.

El $98.19 \%$ de los consumidores encuestados compra jitomate; el $73.06 \%$ compra jitomate Saladet; el $36.6 \%$ compra cada dos días; el 84.87 \% compra de 1 a 2 kg/semana; el $79.34 \%$ compra el jitomate en el mercado local; el $62.73 \%$ lo utiliza para guisar; el 54.98 $\%$ dice que la principal característica de compra que busca es su precio.

El $40 \%$ de los proveedores encuestados traen de Oaxaca capital el jitomate; el 91.94 $\%$ de los consumidores compra Saladet; el $48.39 \%$ vende más de $21 \mathrm{~kg}$ diarios; el 56.45 $\%$ fija el precio del tomate con base en los costos; el $59.68 \%$ se abastece de jitomate cada tercer día. 
Tabla 4. Resultados obtenidos de las encuestas a proveedores en el Istmo oaxaqueño

\begin{tabular}{|c|c|c|c|c|c|c|}
\hline Preguntas & Opciones & $\begin{array}{l}\text { Resultado en } \\
\% \text {, Ixtepec }\end{array}$ & $\begin{array}{l}\text { Resultado en \%, } \\
\text { Tehuantepec }\end{array}$ & $\begin{array}{l}\text { Resultado } \\
\text { en } \% \text {, Salina } \\
\text { Cruz }\end{array}$ & $\begin{array}{l}\text { Resultado en } \\
\text { \%, Juchitán }\end{array}$ & $\begin{array}{l}\text { Total } \\
\text { de los } \\
\text { resul- } \\
\text { tados } \\
\text { en } \% \\
\end{array}$ \\
\hline \multirow{2}{*}{$\begin{array}{l}\text { 1. ¿Produce o compra jito- } \\
\text { mate? }\end{array}$} & Produce & 14 & 0 & 0 & 6 & 4.84 \\
\hline & Compra & 86 & 100 & 100 & 94 & 95.16 \\
\hline \multirow{4}{*}{$\begin{array}{l}\text { 2. Si lo compra, ¿de dónde } \\
\text { es su proveedor? }\end{array}$} & Puebla & 27 & 25 & 30 & 57 & 37.14 \\
\hline & Oaxaca & 39 & 42 & 50 & 30 & 40.00 \\
\hline & Chiapas & 27 & 17 & 10 & 9 & 14.29 \\
\hline & Otros & 7 & 16 & 10 & 4 & 8.57 \\
\hline \multirow{3}{*}{$\begin{array}{l}\text { 3. ¿Cuál es el jitomate que } \\
\text { vende? }\end{array}$} & Criollo & 0 & 8 & 11 & 6 & 6.45 \\
\hline & Saladet & 100 & 92 & 89 & 89 & 91.94 \\
\hline & Bola & 0 & 0 & 0 & 5 & 1.61 \\
\hline \multirow{4}{*}{$\begin{array}{l}\text { 4. ¿Qué cantidad promedio } \\
\text { de jitomate vende al día? }\end{array}$} & $1-10 \mathrm{~kg}$ & 14 & 8 & 17 & 33 & 19.35 \\
\hline & $11-15 \mathrm{~kg}$ & 7 & 17 & 5 & 28 & 14.52 \\
\hline & $16-20 \mathrm{~kg}$. & 29 & 42 & 0 & 11 & 17.74 \\
\hline & $\begin{array}{l}\text { Más de } 21 \\
\mathrm{~kg} .\end{array}$ & 50 & 33 & 78 & 28 & 48.39 \\
\hline \multirow{4}{*}{$\begin{array}{l}\text { 5. ¿Qué factor toma en } \\
\text { cuenta para fijar el precio del } \\
\text { jitomate? }\end{array}$} & Costo & 29 & 92 & 61 & 50 & 56.45 \\
\hline & $\begin{array}{l}\text { Competen- } \\
\text { cia } \\
\end{array}$ & 14 & 0 & 6 & 11 & 8.06 \\
\hline & Calidad & 43 & 8 & 28 & 22 & 25.81 \\
\hline & $\begin{array}{l}\text { Tempo- } \\
\text { rada }\end{array}$ & 14 & 0 & 5 & 17 & 9.68 \\
\hline \multirow{3}{*}{$\begin{array}{l}\text { 6. ¿Cada cuándo se abastece } \\
\text { de jitomate? }\end{array}$} & $\begin{array}{l}\text { Cada } 3 \\
\text { dias }\end{array}$ & 71 & 58 & 61 & 50 & 59.68 \\
\hline & $\begin{array}{l}\text { Cada } \\
\text { semana } \\
\end{array}$ & 29 & 25 & 28 & 11 & 22.58 \\
\hline & Otro & 0 & 17 & 11 & 39 & 17.74 \\
\hline $\begin{array}{l}\text { 7. ¿Cuántos kg de jitomate } \\
\text { compró esta semana? }\end{array}$ & $\begin{array}{l}\text { Kilo- } \\
\text { gramos }\end{array}$ & 2600 & 2720 & 15220 & 9100 & $\begin{array}{l}29 \\
640\end{array}$ \\
\hline
\end{tabular}

Fuente: Elaboración propia. 


\section{Conclusiones}

T $\begin{gathered}\text { os resultados obtenidos } \\ \text { alientan a los empren- } \\ \text { dedores a distraerse de }\end{gathered}$ su producto para focalizarse de manera organizada en la determinación del mercado objetivo y proponer con éxito una estrategia comercial orientada al cliente, con el fin de satisfacer sus demandas y así tener una fidelidad de los clientes.

La interpretación de los resultados permite desarrollar estrategias de comportamientos coordinados de las diferentes funciones de la organización, dirigidas a buscar y amasar información de los consumidores, de la competencia y del entorno. Blesa y Ripollés (2005) señalan que diseminar dicha información por la organización ayuda a diseñar e implementar una respuesta acorde con la información obtenida, basándose para ello en la identificación y construcción de capacidades distintivas de la organización, con el objetivo de satisfacer a los consumidores proporcionándoles un valor superior.

Conocer los resultados obtenidos en las encuestas a los consumidores y proveedores permite amasar información de los consumidores, de la competencia y del entorno para mejorar los productos, reducir errores, defectos y mejorar la productividad con el fin de crear una ventaja competitiva en los mercados. Así se busca alcanzar los estándares de calidad establecidos por los clientes.

Una empresa debe ser capaz de identificar lo que es hoy y lo que desea ser mañana. Debe, además, conocer qué es realmente lo que el cliente desea recibir para diseñar el camino estratégico que le permitirá llegar a la meta. Conocer al cliente es una ventaja competitiva de las empresas, ya que muchas compañías están tomando conciencia de que cada vez son menos rentables las estrategias dirigidas a mercados masivos. Por lo tanto, se vuelve necesario gestionar las relaciones con sus clientes de manera personalizada, desde una perspectiva del valor y de la rentabilidad, a lo largo del ciclo de vida de esas relaciones para obtener una ventaja competitiva sostenible en el tiempo.

La voz del cliente es la principal herramienta con que cuentan las organizaciones, la om describe la lógica de cómo una organización crea, entrega, y captura valor (Osterwalder y Pigneur, 2009). La om se utiliza para describir o diseñar las actividades que necesita o busca la organización para crear valor para los consumidores y otras partes interesadas en el entorno (Wikström et al., 2010).

Las compañías requieren adaptar su infraestructura, procesos y sistemas de gestión para actualizar constantemente el conocimiento sobre sus clientes, analizar su valor y evaluar el intercambio de valor en la relación cliente-empresa. Esto permitirá que las empresas asignen los recursos con prioridad a los clientes más valiosos, puesto que son estos los que dirigen el éxito del negocio.

Los resultados obtenidos permiten a los nuevos emprendedores adaptar los productos a las tendencias del mercado y generar estrategias para tener ventajas competitivas; este proceso de adaptación debe permitir el acceso a nuevas tecnologías y el aprovechamiento de economías de escala, el desarrollo de complementariedades, la planificación de 
acuerdo con las exigencias del mercado y el abaratamiento en los costos por la gestión conjunta de determinados factores de producción. Desde el punto de vista del mercado, este proceso permite adaptarse a la demanda, desarrollar especialidades y mercados propios, cumplir ciertos estándares de calidad y reducir riesgos.

\section{REFERENCIAS BIBLIOGRÁFICAS} Álvarez, L. I., Santos, M. L. y Vázquez, R. (2000). "Análisis cultural y operativo de la orientación al mercado. Efectos moderadores en la relación om-Resultados", Revista Española de Investigación de Marketing-Esic, Vol. 4, No. 1, pp. 7-41.

Anderson, E., Fornell, C., y Mazvancheryl, S. (2004). "Customer Satisfaction and Shareholder Value", Journal of Marketing , 68 (4), 172-185.

Bigné, E., Vila-López, N. y Kuster-Boluda, I. (2000). "Competitive positioning and market orientation: two interrelated constructs", European Journal of Innovation Management, Vol. 3, pp. 190-198.
Blesa, A.y Ripollés M. (2005). "Relación entre la orientación al mercado y la orientación emprendedora: su influencia en el rendimiento de la empresa”. Revista Europea de Dirección y Economía de la Empresa, Vol. 14, núm. 3.

Briggs, R. (2006). "Marketers who measure the wrong thing get faulty answers", Journal of Advertising Research, 462-468.

Deng, S. y Dart, J. (1994). "Measuring market orientation: a multi-factor, multi-item approach", Journal of Marketing Management, Vol. 10, pp. 725-742.

Flavián, C. y Polo, Y. (2001): "Diversidad estratégica y resultados en la distribución", Revista de Economía Aplicada, No. 26 (IX), Otoño, pp. 29-56.

Grant, R. M. (1995). Contemporary strategy analysis: concepts, techniques, applications, Cambridge Massachusetts, US. Basil Blackwell, p. 452.

Grönroos, C. (1989). "Defining marketing: a marketoriented approach", European Journal of Marketing, 23(1), 52-60.
Hernández, R., Fernández, C. y Baptista M. (2010). Metodología de la investigación, quinta edición, Editorial Mc Graw Hill.

Instituto Interamericano de Cooperación para la Agricultura (2010). Desarrollo de los agronegocios y la agroindustria rural en América Latina y El Caribe: Conceptos, instrumentos y casos de cooperación técnica / iica-San José, C. R. ISBN 13: 978-92-9248193-3.

Instituto Nacional de Estadística y Geografía, inegi (2010). http://cuentame. inegi.org. $\mathrm{mx} / \mathrm{monogra}$ fias/informacion/oax/ poblacion/ (consultado en el mes de diciembre del 2012).

Jagpal, S. (2008). Fusion for profit, New York, Oxford University Press.

Kohli, A. y Jaworski, B. (1990). "Market orientation: The construct, research propositions, and managerial implications", Journal of Marketing, Vol. 54, April, pp. 1-18.

Kohli, A., Jaworski, B. \& Kumar, A. (1993). "Markor: A mesure of market orien- 
tation”. Journal of Marketing Research, Vol. 30, November, pp. 467-77.

Kumar, V., Lemon, K. y Parasuraman, A. (2006). "Managing customers for value. An overview and research agenda”, Journal of Service Research, 9 (2), 87-94.

Langerak, F., Peelen, E. y Commandeur, H. (1997). "Organizing for effective new product development", Industrial Marketing Management, Vol. 26, pp. 281-289.

Low, D. (2005). Market orientation, performance and the market environment: a study of Australian manufacturing sme's, anzmac 2005, Conference: Strategic Marketing and Market Orientation, University of Western Sydney, Australia. Disponible en: http:// smib.vuw.ac.nz:8081/ WWW/ANZMAC2005/ index.html

Marketing Science Institute (2004). Research priorities. A guide to msi research programs and procedures, Cambridge, M. A. Author. Matsuno, K., Mentzer, J. T. \& Özsomer (2002). “The effects of entrepreneurial proclivity and market orientation on business performance", Journal of Marketing, Vol. 66, July, pp. 18-32.

Moorman, C., Deshpande, R. y Zaltman, G. (1993). "Factors affecting trust in market research relationship", Journal of Marketing, 58, 81-101.

Morgan, R. M. y Hunt, S. D. (1994). "The commitmenttrust theory of relationship marketing", Journal of Marketing, 58, 20-38.

Morgan, R. E. \& Strong, C. A. (1998). "Market orientation and dimensions of strategic orientation", European Journal of Marketing, 32 (11/129), 10511073.

Narver, J. C. y Slater, S. F. (1990). "The effect of a market orientation on business profitability", Journal of Marketing, Vol. 54, October, pp. 20-35.

Osterwalder, A., \& Pigneur, Y. (2009). Business model generation.

Pelham, A. M. y Wilson, D. T. (1996). "A longitudinal study of the impact of market structure firm structure, strategy, and market orientation culture on dimensions of small firm performance", Journal of the Academy of Marketing Science, Vol. 24, No. 1, pp. 27-43.

Pérez, A. K. (2000). Estrategias de afrontamiento. Diccionario de acción humanitaria, España, 628 pp.

Pinstrup, A. P. (1998). "Una visión global de la agricultura, la alimentación y el medio ambiente". En: Agricultura, medio ambiente y pobreza rural en América Latina. ifpri y bid. pp. 3-20-20.

Reinartz, W., Krafft, M. \& Hoyer, W. (2004). “The customer relationship management process: its measurement and impact on performance", Journal of Marketing Research, 41(3), 293-305.

Porter, M. E. (1985). Competitive advantage: creating and sustaining superior performance, $1^{\text {a }}$ edición, The Free Press, New York.

Ruekert, R. W. (1992): “Developing a market orientation: an organizational strategy perspective", International Journal of Re- 
search in Marketing, Vol. 9 , pp. 225-245.

Samuelson, P. (1983).

Economía, McGraw-Hill de México, 986 pp.

Scaliter, A. (2005). "Método pemo (Primer Estudio de Mercado Objetivo) para nuevos emprendimientos", Revista Temas de Management, de la Universidad del cema. Volumen III.

Sen, A. (1981). Poverty and famines: an essay on entitlement and depression, Oxford University Press.

Subsecretaría de Fomento a los Agronegocios de Oaxaca, sfa (2011). Monitor Agroeconómico para cada uno de los estados de la República Mexicana. Este documento se terminó de elaborar con información al 31 de marzo de 2011.

Stewart, D. (2008). "How marketing contributes to the bottom line", Journal of Advertising Research , 48 (1), 94-105.

Siamwalla, A. y Valdés, A. (1980). "Seguridad alimentaria en los países en vías de desarrollo". En: Desarrollo agrícola en el Tercer Mundo, Carl Eicher y John Staaz (eds.), 1991, fce, México.

Wikström, K., Artto, K., Kujala, J., \& Söderlund, J. (2010). "Business models in project business", International Journal of Project Management, 28, 832-841. Wills, S., \& Webb, S. (2007). "Measuring the value of insight-it can and must be done", International Journal of Market Research, 49 (2), 155-165. 